\title{
Low doses of paclitaxel potently induce apoptosis in human retinoblastoma Y79 cells by up-regulating E2F1
}

\author{
ROSA DRAGO-FERRANTE ${ }^{1}$, ANDREA SANTULLI ${ }^{1,2}$, RICCARDO DI FIORE $^{1}$, MICHELA GIULIANO $^{1}$, \\ GIUSEPPE CALVARUSO $^{1}$, GIOVANNI TESORIERE ${ }^{1 *}$ and RENZA VENTO ${ }^{1 *}$ \\ ${ }^{1}$ Dipartimento di Scienze Biochimiche, Università degli Studi di Palermo, Policlinico, Via Del Vespro 129, 90127 Palermo; \\ ${ }^{2}$ Dipartimento di Scienze Biochimiche, Università degli Studi di Palermo, Sezione di Biochimica Marina, c/o Istituto \\ di Biologia Marina, Consorzio Universitario di Trapani, Lungomare Dante Alighieri, 91016, Trapani, Italy
}

Received April 2, 2008; Accepted May 20, 2008

DOI: 10.3892/ijo_00000053

\begin{abstract}
Paclitaxel (PTX) is an anticancer drug currently in phase II clinical trials. This study shows for the first time that low doses of PTX $(5 \mathrm{nM})$ potently induce apoptosis in human retinoblastoma Y79 cells. The effect of PTX is accompanied by a potent induction of E2F1 which appears to play a critical role in the effects induced by PTX. PTX induced a dose- and time-dependent effect, with $\mathrm{G}_{2} / \mathrm{M}$ arrest, cyclines A, E and B1 accumulation and a marked modification in the status of Cdc2-cyclin B1 complex, the major player of the $G_{2} / M$ checkpoint. Apoptosis followed $G_{2} / M$ arrest. An early and prolonged increase in p53 expression with its stabilization by phosphorylation and acetylation and its nuclear translocation occurred. Consistently, PTX increased p2 ${ }^{\mathrm{WAF} 1}$, bax and MDM2 levels, suggesting that p53 is transcriptionally active. p53 accumulated following both E2F1 up-regulation
\end{abstract}

Correspondence to: Dr Renza Vento, Dipartimento di Scienze Biochimiche, Università degli Studi di Palermo, Policlinico, Via Del Vespro, 90127 Palermo, Italy

E-mail: rvento@unipa.it

${ }^{*}$ Contributed equally

Abbreviations: AO/EB, acridine orange/ethidium bromide; BSA, bovine serum albumin; $\mathrm{DiOC}_{6}$, 3,3-dihexyloxacarbocyanine; DMSO, dimethyl sulfoxide; $\Delta \psi_{\mathrm{m}}$, mitochondrial transmembrane potential; FasR, Fas receptor; FasL, Fas ligand; GAPDH, glyceraldehyde 3-phosphate dehydrogenase; JNK, Jun N-terminal kinase; MDM2, mouse double minutes 2; MTT, 3-(4,5-dimethylthiazol2-yl)-2,5-diphenyltetrazolium bromide; PARP, poly (ADP-ribose) polymerase; PBS, phosphate-buffered saline; PCR, polymerase chain reaction; PFT- $\alpha$ pifithrin- $\alpha$; pRb, retinoblastoma protein; PTX, paclitaxel; SP600125, anthra [1,9-cd]pyrazol-6(2H)-one-1,9pyrazoloanthrone; z-VAD-fmk, benzyloxy carbonyl-Val-Ala-Aspfluoromethylketone

Key words: retinoblastoma, paclitaxel, apoptosis, E2F1, pRb and increase in the levels of p14 ${ }^{\mathrm{ARF}}$ which interacts with MDM2 preventing ubiquitination and proteosomal degradation of $\mathrm{p} 53$. Both extrinsic (E2F1/Fas/JNK/caspase-2 activation) and intrinsic (Bcl-2 phosphorylation, Bid fragmentation and Bax increase) pathways seemed to be involved. Loss of mitochondrial potential and activation of apoptosome and executive caspase-3,-6 and-7 was shown. Incubation with either the irreversible pan-caspase inhibitors Z-VADFMK, or SP600125, a selective inhibitor of JNK, or pifithrin $\alpha$, a potent p53 inhibitor, significantly inhibited the effects induced by PTX.

\section{Introduction}

Retinoblastoma is the most common eye cancer affecting children (0-5 years). Children with the inherited form have a high risk of developing other cancers later in life (1). If untreated, the patients die of intracranial extension and disseminated disease within two years. Though chemotherapy with adjuvant local treatments is adequate for early tumors, enucleation still plays a major role in the treatment of retinoblastoma (2). Recently, repeated cycles of chemotherapy with carboplatin, vincristine and etoposide combined with cryotherapy and laser photocoagulation have improved the ocular salvage rate for children with bilateral retinoblastoma (3). However, children with late-stage disease often experience recurrence after treatment. In addition, as ototoxicity is a possible side-effect of carboplatin (4), the evaluation of hearing loss in patients which frequently have impaired vision is very important.

Retinoblastoma is caused by mutational inactivation of the tumor suppressor RB, the only known human gene whose mutation is sufficient for a human cancer (5). In addition to causing retinoblastoma, deregulation of $\mathrm{RB}$ contributes to most other types of human cancer (6). The retinoblastoma protein $(\mathrm{pRb})$ is the most investigated tumor suppressor, and the interest in this protein springs essentially from its ability to slow down or abrogate neoplastic growth. pRb is responsible for a major G1 checkpoint, blocking S-phase entry by targeting E2F transcription factors (7). Alterations in the $\mathrm{pRb} / \mathrm{E} 2 \mathrm{~F}$ pathway are commonly found in human cancers 
and in the absence of $\mathrm{pRb}$, multiple pathways are activated leading to increased tumors (8).

During recent years the overall goal of our laboratory was to gain a better understanding of how $\mathrm{pRb}$ alteration induces the onset of cancer (9-12). Our studies, performed in human retinoblastoma Y79 cells, have shown that in these cells the lack of functional $\mathrm{pRb}$ is accompanied by upregulation of p53 and this renders Y79 cells prone to apoptosis (13). We have demonstrated that in these cells a number of drugs act as apoptosis-inducers through either extrinsic or intrinsic pathways $(14,15)$, via p53-dependent (16) or p53-independent (17) mechanisms, with some drugs inducing significant synergistic effects when employed in combination (18-20).

The search for drugs that are non-cytotoxic to normal cells and can effectively target cancer cells, has led to investigation of the potential anti-cancer activity of some natural compounds, such as Paclitaxel (PTX) which belongs to a novel class of anticancer drugs (taxanes), extracted from Taxus species (21). PTX is an antimicrotubule chemotherapy agent that, owing to its unique structure, readily enters mammalian cells and preferentially binds to tubulin in polymerized microtubules. Microtubule dynamic is crucial to many important cellular functions, and during mitosis they are at their highest dynamic instability to allow the assembly of a functional bipolar spindle for the passage through the metaphase/ anaphase checkpoint, during which chromosomes are aligned and segregate. The action of PTX results in the formation of stable bundles of microtubules within the cell, with inhibition of mitotic spindle, mitotic and $\mathrm{G}_{2}-\mathrm{M}$ arrest and apoptosis (22). Although PTX is known to be cytotoxic against several tumor cell lines, including advanced human carcinomas refractory to traditional chemotherapy, and this ability to harness microtubules for killing cancer cells has yielded therapies for multiple solid tumors, such as breast, ovarian, bronchogenic, colon, and other carcinomas (23), whether such a strategy could also be effective in retinoblastoma cells is unknown. Since pRb disruption can accelerate $\mathrm{G} 1$ to $\mathrm{S}$ and $\mathrm{S}$ to $\mathrm{G}_{2} / \mathrm{M}$ progressions, we hypothesized that PTX, known to induce $\mathrm{G}_{2}-\mathrm{M}$ arrest, could be particularly effective in activating apoptosis in Y79 cells that lack functional $\mathrm{pRb}$. Here we demonstrate for the first time that PTX potently induces apoptosis in human Y79 retinoblastoma cells. Apoptosis followed up-regulation of E2F1, accumulation of cyclin E, cyclin A and cyclin B1, changes in the functional status of $\mathrm{Cdc} 2$ and $\mathrm{G}_{2}-\mathrm{M}$ arrest. The effects were determined by very low concentrations of PTX ( $5 \mathrm{nM})$ and were accompanied by potent up-regulation of $\mathrm{p} 53$, significant increases in the levels of mouse double minutes 2 (MDM2), the predominant negative regulator of p53, (24) and potent increase in $\mathrm{p} 14^{\mathrm{ARF}}$, the inhibitor of $\mathrm{p} 53-\mathrm{MDM} 2$ interaction (25). Fas Receptor (FasR), Fas Ligand (FasL) and c-Jun amino terminal kinase/stress-activated protein kinase (JNK) also appeared to be implicated. The activation of initiator and executioner caspases and the involvement of Bcl-2 family and mitochondrial pathway were shown. To our knowledge, this is the first study demonstrating that PTX upregulates E2F1. Its potent apoptotic effect on human Y79 retinoblastoma cells should be considered for clinical investigation.

\section{Materials and methods}

Cells, reagents and solutions. Human retinoblastoma Y79 cells were purchased from Interlab Cell Line Collection, Genova, Italy. The JNK inhibitor SP600125 (anthra [1,9cd]pyrazol-6(2H)-one-1,9-pyrazoloanthrone), the selective inhibitors for p53 (pifithrin- $\alpha$, PFT- $\alpha$ ), heat-inactivated fetal bovine serum (FBS), L-glutamine $(200 \mathrm{mM})$, penicillin/ streptomycin $(10,000 \mathrm{IU} / \mathrm{ml}$ and $10,000 \mathrm{mg} / \mathrm{ml}$, respectively), Hoechst stain solution No. 33258 (2-2(4-hydroxyphenol)6-benzimidazolyl-6-/1-methyl-4-pierpazyl)-lbenzimidaloltrihydrochloride, acridine, ethidium bromide, 3-(4,5-dimethylthiazol-2-yl)-2,5-diphenyltetrazolium bromide (MTT), Dimethyl sulfoxide (DMSO) and paclitaxel (PTX) were purchased from Sigma (St. Louis, MO). The general caspase inhibitor z-VAD-fmk (Benzyloxy Carbonyl-Val-Ala-Aspfluoromethylketone) was purchased from Promega (Milan, Italy). Plastic dishes were obtained from Corning-Costar (Cambridge, MA) and all other tissue culture plastics were from Falcon Plastics (Los Angeles, CA). Chamber Slide System was purchased from Lab Tek (Nalge Nunc International). Unless otherwise specified, all antibodies were purchased from Santa Cruz Biotechnology, Inc. (Santa Cruz, CA). The secondary horseradish peroxidase-labeled goat anti-mouse and goat anti-rabbit antibodies were from Amersham Life Science Inc. (Arlington Heights, IL). Antiphospho cdc2 (Tyr15), anti-cdc2 and antibodies against cyclin B1 were obtained from Cell Signaling (Beverly, MA). Stock solution of PTX were $4 \mathrm{mM}$ in DMSO, protected from light and stored at $-20^{\circ} \mathrm{C}$. Just prior to PTX treatment, the stock solution was freshly diluted in RPMI-1640 medium. Final concentrations of DMSO never exceeded $0.04 \%$. Stock solutions of z-VAD-fmk (100 mM) were prepared in DMSO. When indicated, $\alpha$-pifithrin was applied $1 \mathrm{~h}$ prior to treatment with PTX. Protein concentration was assayed using the method of Lowry (26).

Cell culture and paclitaxel treatments. Human retinoblastoma Y79 cells were grown in suspension in RPMI-1640 and $15 \%$ FBS as previously described (13). Media were changed every 2-3 days. For monolayer cultures Y79 cells were seeded on 96 -well $\left(5 \times 10^{4}\right.$ cells $/ 0.2 \mathrm{ml} /$ well $)$ or 6 -well plates $\left(1.5 \times 10^{6}\right.$ cells $/ 4 \mathrm{ml} /$ well $)$, precoated with $5 \mu \mathrm{g} / \mathrm{cm}^{2}$ poly-D-lysine. Twenty-four hours after seeding, cells were treated with PTX for the time indicated in the Results. For studying the concentration- and time-dependent effects, Y79 cells were treated with 0.5-10 nM PTX for 4-96 h. In the other experiments, Y79 cells were treated with $5 \mathrm{nM}$ PTX. Control group was treated with the same amount of DMSO employed as vehicle for PTX treatment.

MTT colorimetric survival assay. Cell viability was determined by the MTT colorimetric assay (27) as previously described (17). Dye absorbance in viable cells was measured at $570 \mathrm{~nm}$, with $630 \mathrm{~nm}$ as a reference wavelength. Cell survival was estimated as a percentage of the value of the vehicle-treated control. All experiments were repeated at least six times, and each experimental condition was repeated at least in quadruplicate wells in each experiment. The direct proportion between dye reduction and cell number extends 
from $1 \times 10^{3}$ to $5 \times 10^{5}$ cells/well. In some experiments trypan blue was used to count viable cells with comparable results.

Cell death. To study cell death parameters the following experiments combining different techniques were performed: a) Hoechst 33258 staining: Y79 cells were seeded on sterile chamber slides and allowed to attach. Cells were then treated with PTX for the indicated times at $37^{\circ} \mathrm{C}$, then cells were fixed with methanol/acid acetic $(3: 1)$ for $10 \mathrm{~min}$ at room temperature. After rinsing with PBS, the slides were incubated for $10 \mathrm{~min}$ at room temperature in Hoechst 33258 $(5 \mu \mathrm{g} / \mathrm{ml})$ and then washed three times in PBS prior to examining under a Leica fluorescent microscope equipped with an automatic photomicrograph system; b) Acridine Orange (A/O)-Ethidium Bromide (EB) staining: morphological evidence of apoptosis was also obtained by means of $\mathrm{AO} / \mathrm{EB}$ staining. Monolayer cultures in 96-well plates were employed for these studies. After removal of the incubation medium, cells were rinsed and treated with a solution composed of AO/EB (100 $\mu \mathrm{g} / \mathrm{ml}$ PBS of each dye). Cells were then observed with fluorescent microscope. The emission wavelength were set at 525 and $610 \mathrm{~nm}$; and c) cell cycle analysis by flow cytometry: after PTX treatment, cells were harvested and centrifuged. Then, the cells were fixed with cold ethanol, resuspended in hypotonic solution containing $10 \mu \mathrm{g} / \mathrm{ml}$ propidium iodide, $0.1 \%$ sodium citrate, $0.01 \%$ Nonidet P-40 and $100 \mu \mathrm{g} / \mathrm{ml}$ RNase A and incubated for $1 \mathrm{~h}$ at room temperature. Cell cycle phase distribution was evaluated by an Epics XL flow cytometer (Beckman Coulter) using Expo32 software. The percentage of cells in the subdiploid region was considered as an index of apoptosis.

Measurement of mitochondrial transmembrane potential $\left(\Delta \psi_{m}\right)$. Mitochondrial transmembrane potential $\left(\Delta \psi_{\mathrm{m}}\right)$ was measured by using 3,3-dihexyloxacarbocyanine ( $\mathrm{DiOC}_{6}$ Molecular Probes, Eugene, OR), a fluorochrome which exclusively emits within the spectrum of green light and accumulates in the mitochondrial matrix under the influence of $\Delta \psi \mathrm{m}$. Cells were incubated with $40 \mathrm{nM} \mathrm{DiOC}_{6}$ for $20 \mathrm{~min}$ at $37^{\circ} \mathrm{C}$, washed twice with PBS and analysed by flow cytometry on Beckman Coulter Epics XL flow cytometer with excitation and emission settings of 488 and $525 \mathrm{~nm}$, respectively. The percentage of cells showing a lower fluorescence, reflecting loss of mitochondrial transmembrane potential, was determined by comparison with untreated control using Expo32 software. Carbonyl cyanide m-chlorophenylhydrozone $(\mathrm{CCCP}, 50 \mu \mathrm{M})$, a protonophore that completely de-energizes mitochondria by dissipating the transmembrane potential, was used as a positive control for maximum $\Delta \psi_{\mathrm{m}}$ disruption.

Analysis of microtubule arrays by immunofluorescence microscopy. Cells attached to poly(D-lysine)-coated chamber slide were treated with PTX for various times. Thereafter, cells were fixed with 3\% paraformaldeide in PBS for 2 min at room temperature (RT) and washed 3 times with PBS. Then, cells were permeabilized with $1 \%$ Triton $\mathrm{X}-100$ for $1 \mathrm{~min}$ at RT and washed 3 times with PBS. Non-specific sites were blocked by incubating with 5\% skim BSA in PBS for $1 \mathrm{~h}$. Mouse monoclonal antibody against $\beta$-tubulin was diluted
1:500 in blocking solution and incubated for $2 \mathrm{~h}$ at RT. Cells were washed with PBS to remove antibody excess and probed with FITC-conjugated secondary antibody (1:200) for $1 \mathrm{~h}$ at RT. The images of $\beta$-tubulin with FITC staining were visualized by fluorescence microscopy and captured with Leica Q-Fluoro.

Assay to measure the cytoplasmic-nuclear shuttling of p53. Cells were fixed with methanol/acid acetic (3:1) for 10 min, washed with PBS and incubated with Hoechst 33258 $(5 \mu \mathrm{g} / \mathrm{ml})$ in PBS for $10 \mathrm{~min}$. Thereafter, the cells were washed with PBS and incubated with antibody anti-p53 (1:500) for $1 \mathrm{~h}$. After washing with PBS to remove antibody excess, the cells were probed with FITC-conjugated secondary antibody (1:200) for $1 \mathrm{~h}$ at room temperature. The images were visualized by fluorescence microscopy and captured with Leica Q-Fluoro.

Western blot and immunoprecipitation. After treatment, Y79 cells were washed with PBS and incubated in ice-cold lysis buffer containing protease inhibitor cocktail for $20 \mathrm{~min}$, as previously reported (13). Then, cells were sonicated three times for $10 \mathrm{sec}$. Equal amounts of protein samples $(60 \mu \mathrm{g} /$ lane) were subjected to SDS-polyacrylamide gel electrophoresis and then transferred to a nitrocellulose membrane for detection with specific antibodies. The correct protein loading was verified by means of both red Ponceau staining and immunoblotting for actin.

For immunoprecipitations, $400 \mu \mathrm{g}$ of nuclear extracts was incubated with $0.4 \mu \mathrm{g}$ of the appropriate primary antibody for $3 \mathrm{~h}$ and then incubated overnight with $30 \mu \mathrm{l}$ of protein $\mathrm{A} / \mathrm{G}$ plus agarose beads. The immunocomplexes were washed with lysis buffer, boiled in SDS sample buffer and submitted to Western blotting. Mock immunoprecipitations in the absence of antibody were performed.

RT-PCR analysis. Total RNA was extracted from Y79 cells using RNeasy Mini Kit (Qiagen) and treated with DNase I using RNase-freeDNase set (Qiagen) to eliminate genomic DNA. RNA $(1 \mu \mathrm{g})$ was reverse transcribed using Moloney murine leukemia virus reverse transcriptase (Promega, USA) and cDNA was amplified in $25 \mathrm{ml}$ of PCR reaction mixture as previously reported (8). Amplified products were resolved by agarose gel electrophoresis ( $1 \%$ agarose $-0.5 \mu \mathrm{g} / \mathrm{ml}$ ethidium bromide) and the gel image was acquired with the Quantity One Software. GAPDH was employed as an internal control. The following primer pairs (Proligo USA) were used: GAPDH (200 bp): sense, 5'-TGACATCAAGAAGGTGA-3'; antisense, 5'-TCCACCACCCTGTTGCTGTA-3'; Fas-L (500 pb): sense, F,5'-ATGTTTCAGCTCTTGCACCTACAG3'; antisense, 5'-TGCATAGACTCGAGAGAGACCA-3'; Fas-R (200 pb): sense, 5'-TTCACTTCGGAGGATTGCTC-3'; antisense, 5'-GCTTTATGGCAGAATTGGCC-3'; p21 (160 $\mathrm{pb})$ : sense, 5'-AGGGGACAGCAGAGGAAG-3'; antisense, 5'-CGGCGTTTGGAGTGGTAG-3'.

\section{Results}

Paclitaxel impaires cell viability, changes cell morphology and induces apoptosis in Y79 cells. To examine the effects of 
paclitaxel (PTX) on Y79 cells, monolayer cultures were treated for 4-96 h with various doses $(0.2-10 \mathrm{nM})$ of the drug and viability was studied by MTT assay to measure mitochondrial enzyme activity. Fig. 1A shows that viability decreased with the time in a dose-dependent manner and that the effect resulted in an almost maximum decrease at $5 \mathrm{nM}$, a concentration that reduced cell viability by $78 \%$ after 48-h treatment. Viability was also routinely evaluated by trypan blue staining (not shown), with similar results. Thus, 5 nM PTX was employed throughout the present study.

In Fig. 1B, light microscopy demonstrates that $5 \mathrm{nM}$ PTX progressively reduced the cell number and also induced dramatic modifications in cell shape, with a doubling of their dimension seen already after 8 -h treatment and markedly increasing after $16 \mathrm{~h}$. Since this treatment-time, most cells rounded up losing contact with neighboring cells and most cells appeared apoptotic as suggested by membrane blebbing, apoptotic bodies formation and a tendency to float in the medium. As chromatin condensation and nuclear fragmentation remain the hallmarks of apoptotic cells, apoptosis was assessed by nucleic acid staining with Hoechst 33258 (Fig. 1C), which revealed that after $16 \mathrm{~h}$ of treatment PTX induced typical apoptotic nuclei exhibiting highly fluorescent condensed chromatin without any discernible structure. The complementary, semiquantitative fluorescent dye technique by using AO/EB staining (Fig. 1D) confirmed that Y79 cells treated with PTX exhibited typical morphological changes of apoptosis with nuclear chromatin condensation induced in a time-dependent manner (16-48 h). Percentages of apoptotic cells, estimated on the basis of AO/EB staining, corresponded well with the loss in cell viability measured by MTT assay.

Paclitaxel affected microtubule organization and induced accumulation of cyclines with $G_{2} / M$ phase arrest followed by accumulation of the cells in the sub $G_{0} / G_{1}$ phase. It is well known that in several cell lines PTX binds to and stabilizes microtubules leading to mitotic arrest of the cell cycle and subsequent apoptosis. Here, we exhamined whether also in Y79 cells PTX treatment affected the cellular microtubule network. In Fig. 2A, immunofluorescence microscopy employing anti-tubulin antibody shows that in control cells microtubule network exhibited normal organization with a delicate arrays of short microtubules at the perinuclear membrane. PTX-treatment resulted in microtubule polymerization with formation of long thick microtubule bundles surrounding the nucleus; the effect occurred as early as after $2 \mathrm{~h}$ and increased with time peaking at $16-24 \mathrm{~h}$; in cells that displayed doubled dimension, microtubule asters were evident. To determine whether microtubule polymerization induced by PTX treatment was accompanied by mitotic arrest, we performed cytofluorimetric analysis in the presence of propidium iodide, in order to quantify the percentage of cell distribution troughout cell cycle phases. Fig. $2 \mathrm{~B}$ shows that $\sim 62 \%$ of control cells were in the $G_{0} / G_{1}$ phase and $\sim 17 \%$ were in the $G_{2} / M$ phase. No subG $G_{0} / G_{1}$ phase (apoptosis) was detected. PTX induced a time-dependent accumulation of the cells in the $\mathrm{G}_{2} / \mathrm{M}$ phase with concomitant loss from $\mathrm{G}_{0}-\mathrm{G}_{1}$ phase. After 2 and 4 h of treatment $\sim 21 \%$ and $\sim 25 \%$ of cells, respectively, accumulated in the $\mathrm{G}_{2} / \mathrm{M}$ phase; accumulation increased with time reaching the maximum at $16 \mathrm{~h}(\sim 58 \%)$ and $24 \mathrm{~h}(\sim 60 \%)$ and declining thereafter ( $\sim 33 \%$ after $48 \mathrm{~h}$ of treatment). The figure also shows that until $8 \mathrm{~h}$ of PTX treatment little or no events were recorded in the sub $\mathrm{G}_{0} / \mathrm{G}_{1}$ phase; a characteristic sub- $\mathrm{G}_{0} / \mathrm{G}_{1}$ peak was detected after $16 \mathrm{~h}(\sim 9 \%)$ and markedly increased after $24 \mathrm{~h}(\sim 23 \%)$, and $48 \mathrm{~h}(\sim 59 \%)$ of treatment. These results strongly suggest that the onset of apoptosis induced by PTX followed $\mathrm{G}_{2} / \mathrm{M}$ phase arrest.

Cyclin-dependent kinases and cyclins are well known regulators of the eukaryotic cell cycle, with cyclin $\mathrm{E}$ and cyclin A promoting cell cycle progression through $\mathrm{G} 1 / \mathrm{S}$ transition and with cyclin $\mathrm{B} 1$ and $\mathrm{Cdc} 2$ regulating progression from the $\mathrm{G}_{2}$ phase into mitosis .

Western blot analysis of the $\mathrm{G}_{2}-\mathrm{M}$ regulatory proteins (Fig. 2C) shows that the $\mathrm{G}_{2}-\mathrm{M}$ arrest induced by PTX was associated with a progressive accumulation of cyclin A and cyclin E from 4 to $48 \mathrm{~h}$ of treatment, with cyclin A expressing much higher levels than cyclin E. The figure also shows that PTX led to potent, inappropriate accumulation of cyclin B1 throughout the treatment. Part of the mechanism by which PTX blocks cells at the $\mathrm{G}_{2} / \mathrm{M}$ checkpoint seems to involve the regulation of Cdc2 activity by its reversible phosphorylation/ dephosphorylation at Thr-14 and Tyr-15, which suppresses/ activates its kinase activity. In our experiments PTX treatment induced a biphasic effect on both Cdc2 levels and phosphorylation. In Fig. 2D, after 4, 8 and $16 \mathrm{~h}$ of treatment Cdc2 protein decreased below its basal levels, while its inactive Tyr15-phosphorylated-Cdc2 form markedly increased. Successively, after 24 and 48 h of treatment, Cdc2 levels significantly increased again, over control and this was accompanied by a potent decrease in the inactive Tyr15phosphorylated-Cdc2 form.

PTX increased E2F1 levels early followed by p53 increase, stabilization and activation. It is known that $\mathrm{pRb}$ loss upregulates E2F1 levels which could cooperate with p53 in promoting apoptosis through direct interaction. In Fig. 3A, Western blot analysis shows that control Y79 cells express measurable basal levels of E2F1. Treatment with PTX progressively increased E2F1 levels. The increase was already observed after $30 \mathrm{~min}$ of treatment and peaked at 8-48 h. The same figure shows that E2F1 induction was accompanied by up-regulation of p53 which increased between 2 and $48 \mathrm{~h}$ of treatment. The time course of p53 accumulation by PTX treatment shows that $\mathrm{p} 53$ increase occurred before the onset of apoptosis and suggests that it could be under the action of E2F1. To establish whether E2F1 can bind p53, immunoprecipites obtained by anti-E2F1 antibody, were reacted with either anti E2F1 or anti p53-antibodies (Fig. 3B). The figure shows that E2F1 and p53 interacted, and that the interaction was higher after PTX treatment, accordly with the levels reached by each partner after PTX treatment. Consistently, immunoprecipitates obtained by anti-p53 antibody potently reacted with anti-E2F1 antibodies (data not shown).

Analysis by immunofluorescence of p53 (Fig. 3C), shows that PTX induced a potent increase in p53 levels. The effect was evident after $2 \mathrm{~h}$ of treatment and increased until $16 \mathrm{~h}$. Fig. 3C also suggests that p53 accumulates to the nucleus, as the progressive p53 localization to the Hoechst-positive nuclear material (merge) demonstrates (Fig. 3D). The figure 

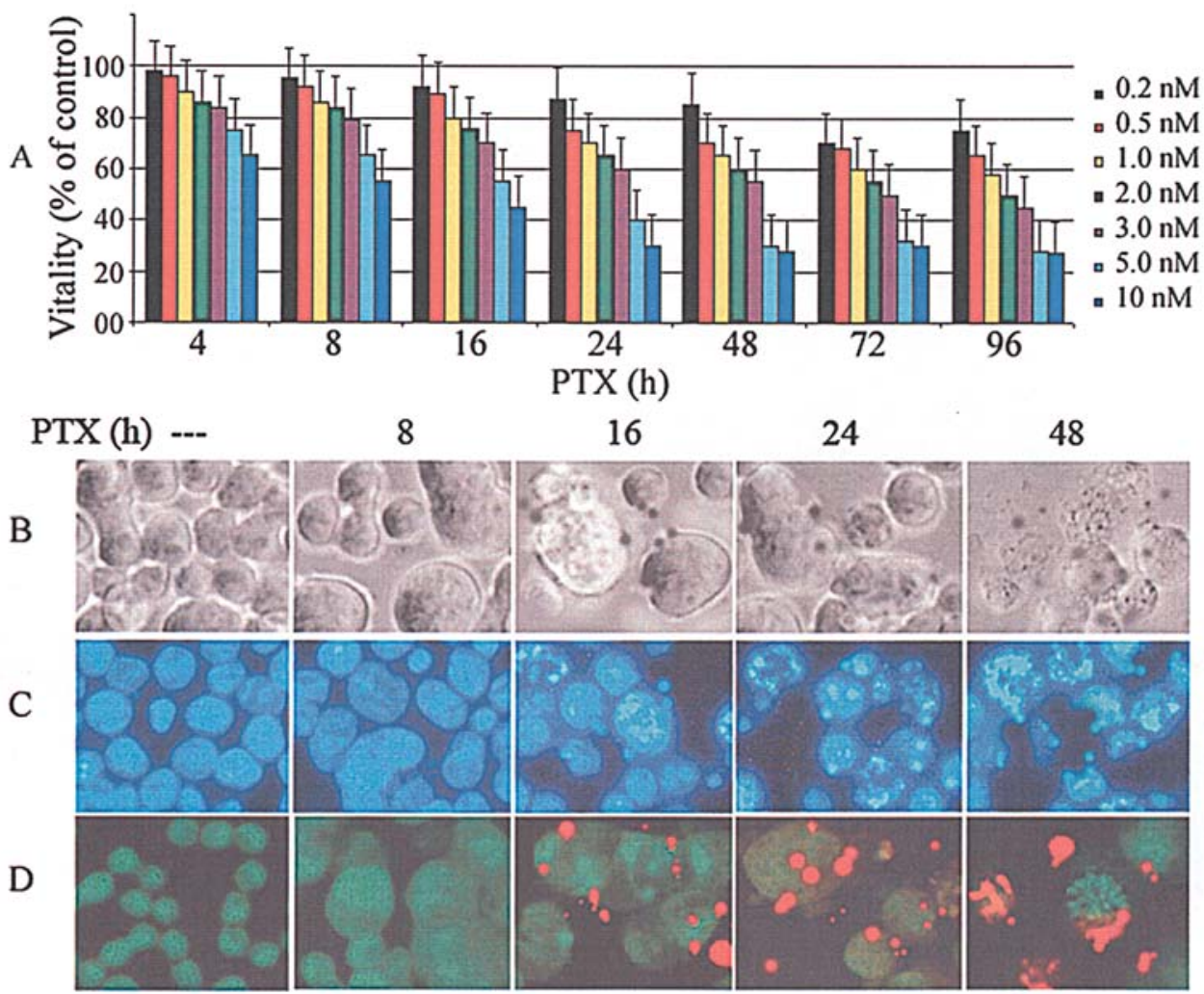

Figure 1. Paclitaxel impaired Y79 cell viability, changed cell dimensions and induced apoptosis. (A) Y79 cells were treated with 0.2-10 nM of PTX for 4-96 h and studied for MTT assay as described in Materials and methods. Data are mean \pm SEM of six independent experiments each carried out in quadruplicate. For microscopy analysis, Y79 cells were treated with $5 \mathrm{nM}$ PTX for the indicated times and examined as described in Materials and methods by: (B) light microscopy; (C) fluorescence microscopy by Hoechst 33258 staining; (D) fluorescence microscopy by acridine orange/ethidium bromide staining. (x400 magnification). Fluorescent nuclei were screened for normal morphology (unaltered chromatin), and apoptotic nuclei (with fragmented and condensed chromatin)

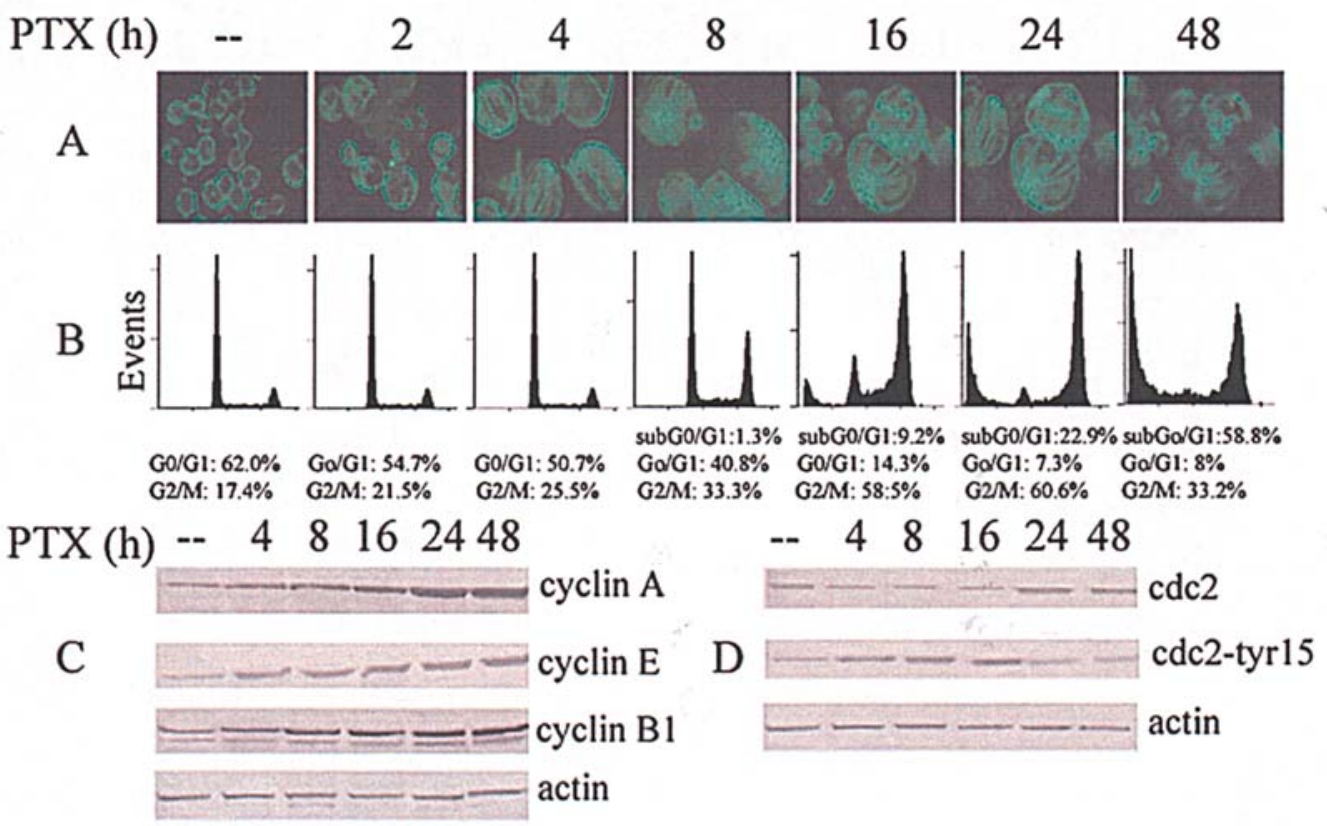

Figure 2. Paclitaxel induced microtubule polymerization, cell accumulation in $\mathrm{G}_{2} / \mathrm{M}$ phase and increases in cyclin levels. Y79 cells were treated with $5 \mathrm{nM}$ PTX for the indicated times and analized as described in Materials and methods by: (A) immunofluorescence microscopy with anti- $\beta$-tubulin antibodies to describe the microtubule arrays. The normal organization of short microtubule network at the perinuclear membrane is seen in control cells (without PTX treatment). After PTX-treatment cells display doubled dimension and long polymerized microtubule bundles; (B) flow cytometry with propidium iodide to describe cell cycle profiles. The time of $\mathrm{G}_{2} / \mathrm{M}$ phase accumulation and the sub $\mathrm{G}_{0} / \mathrm{G}_{1}$ peak induced by PTX treatment agree well with microscope observations. (C and D) Western blot analysis to describe the status of $\mathrm{G}_{2} / \mathrm{M}$ regulator proteins. The results reported in A-D panels are representative of at least four independent experiments. 
A

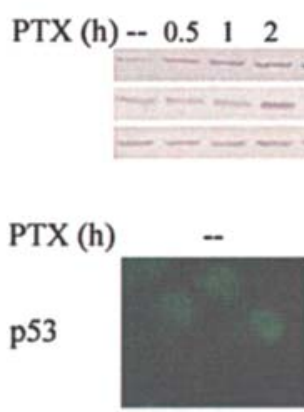

PTX (h)

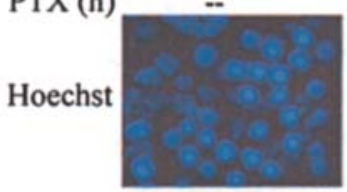

p53



Merge
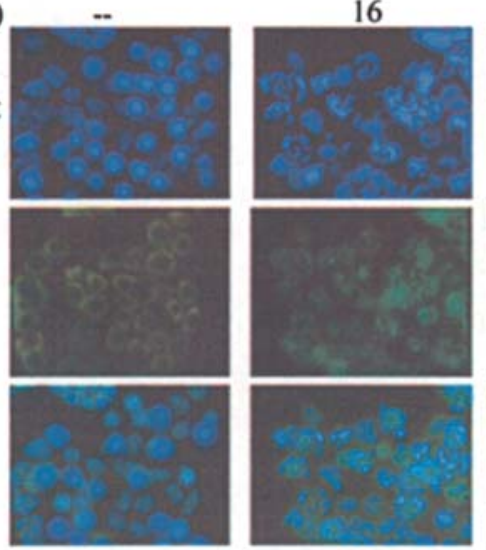

24
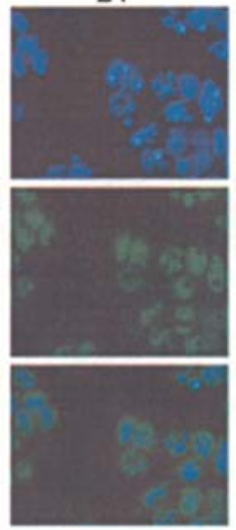

B

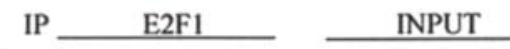

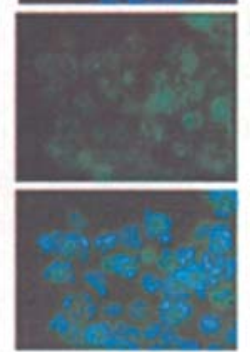

4 8

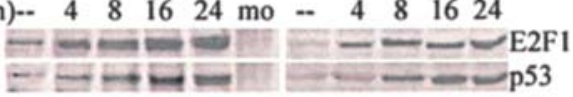

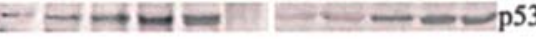

$8 \quad 16$

24
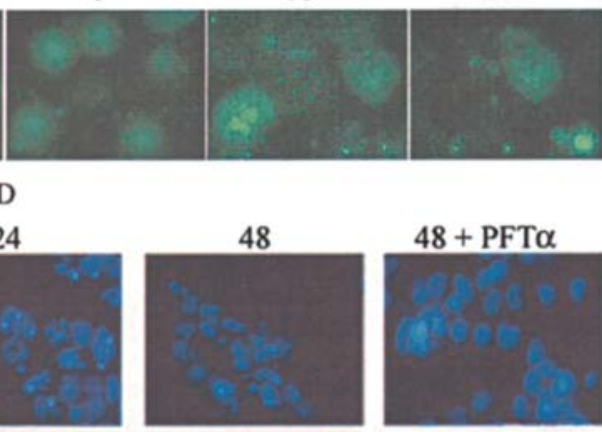

$48+$ PFT $\alpha$
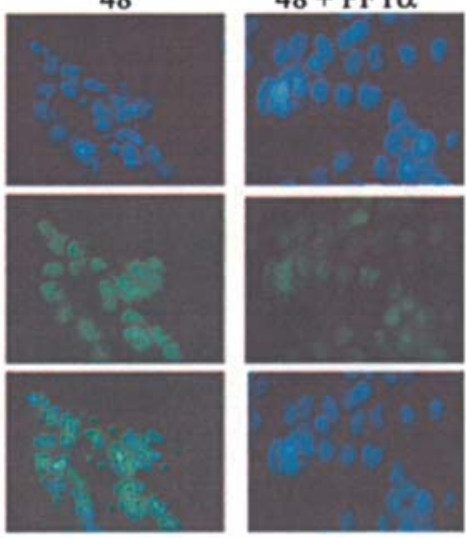

Figure 3. Y79 cells express high levels of E2F1 which increases with PTX treatment. PTX also induced both p53 increases and nuclear translocation. Y79 cells were treated with $5 \mathrm{nM}$ PTX for the indicated times and examined as described in Materials and methods by: (A) Western blot analysis to describe the levels of both E2F1 and p53; (B) immunoprecipitation analysis to describe the levels of E2F1/p53 interaction. In (B) input, 15\%; mo, mock; (C) immunofluorescence microscopy to describe nuclear accumulation of p53. (D) Immunofluorescence microscopy to describe cytosol/nuclear shuttle of p53 by Hoechst 33258 staining (top panels) which shows the progressive DNA fragmentation induced by PTX treatment, and immunofluorescence analysis (middle panel) which shows the progressive nuclear accumulation of p53. In the bottom panel, the p53 localization to the Hoechst-positive nuclear material (merge) is shown. Right panels show the effects of pre-treatment with Pifithrin- $\alpha$ (PFT- $\alpha$ ), an inhibitor of p53 activity. The results reported in A-D panels are representative of at least 5 independent experiments.

A

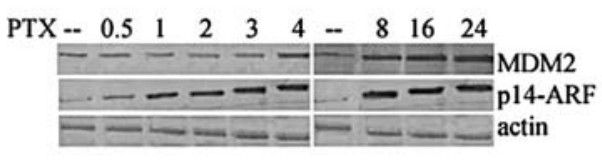

C

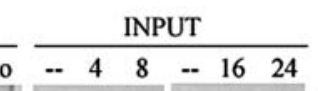

\begin{tabular}{|c|c|c|c|}
\hline--4 & 8 & $\begin{array}{lll}-- & 1624\end{array}$ & \\
\hline & & & p53ser15 \\
\hline & & & p53lys373 \\
\hline & & & \\
\hline
\end{tabular}

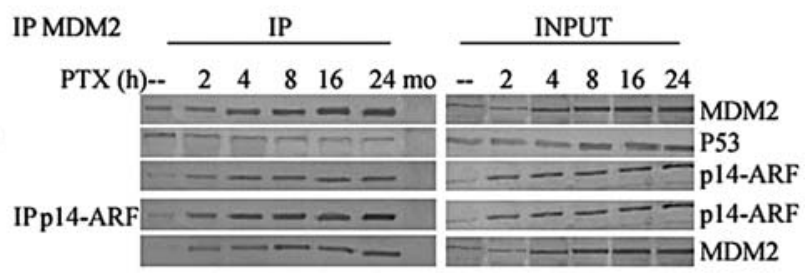

D

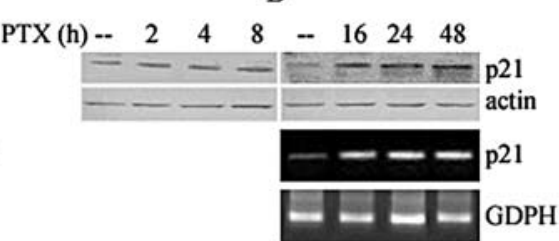

Figure 4. Effects of PTX on MDM2 and p14-ARF levels and on p53 stabilization. Y79 cells were treated with $5 \mathrm{nM}$ PTX for the indicated times and examined as described in Materials and methods by: (A) Western blot analysis to describe the levels of both MDM2 and p14 ARF; (B and C) immunoprecipitation analysis to describe the MDM2/p53/p14 $\mathrm{ARF}$ interaction. In (B and C) input, 15\%; mo, mock; (D) Western blot and RT/PCR analysis to describe $\mathrm{p} 21$, one of the transcriptional targets of p53. The results reported in A-D panels are representative of at least 3 independent experiments.

also shows (right panels) that pretreatment of Y79 cells with Pifithrin- $\alpha$ (PFT- $\alpha)$, a potent inhibitor of p53 activity, significantly decreased the effect induced by PTX on nuclear p53 translocation. 
A

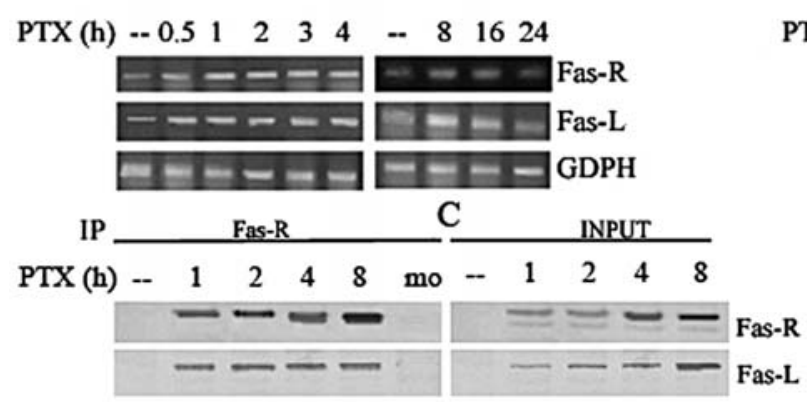

D

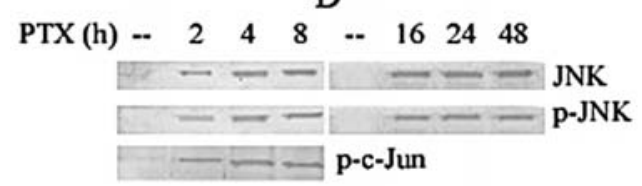

B



E

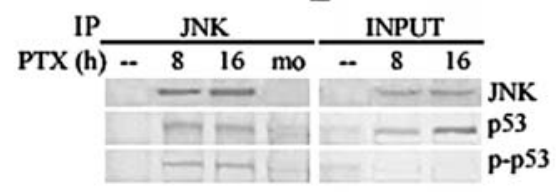

Figure 5. Involvement of FasR, FasL and JNK in PTX induced apoptosis. Y79 cells were treated with 5 nM PTX for the indicated times and examined as described in Materials and methods by: (A) RT/PCR to describe the levels of FasR and FasL mRNA; (B and D) Western blotting to describe the levels of FasR, FasL, JNK, pJNK and p-cJun protein; (C and E), immunoprecipitation analysis to describe FasR/FasL interaction and JNK/ p53 interaction. In (C and E) input, $15 \%$. mo, mock. The results reported in panels A-E are representative of at least four independent experiments.

To evaluate if p53 level is controlled by its negative regulator MDM2, known to target p53 for proteasomal degradation, the levels of this protein was studied during the time of PTX treatment. In Fig. 4A, Western analysis shows that PTX induced a progressive increase in the level of MDM2 from 4 to $24 \mathrm{~h}$ of treatment. In addition, and in a time which correlates well with E2F1 induction, PTX markedly increased the level of p14 ${ }^{\mathrm{ARF}}$. In Fig. 4B immunoprecipitates obtained by anti-MDM2 antibodies were reacted with anti-MDM2 or with anti-p53 or with anti-p14 ${ }^{\mathrm{ARF}}$ antibodies. The figure shows that in control cells p53 interacted well with MDM2; after PTX treatment, in spite of p53 accumulation, p53-MDM2 interaction appeared weaker, instead MDM2/p14 ${ }^{\mathrm{ARF}}$ interaction increased according to p14 ${ }^{\mathrm{ARF}}$ accumulation. The same figure shows that immunoprecipitates obtained by anti-p14 ${ }^{\mathrm{ARF}}$ antibodies, after PTX treatment, markedly reacted with anti-MDM2 antibodies. The results strongly suggest that $\mathrm{p} 14^{\mathrm{ARF}}$ relieves $\mathrm{p} 53$ from MDM2 inhibition.

Since the key to the magnitude and duration of p53 activities lies in its stability, which depends on its phosphorylation and/or acetylation, we evaluated whether PTX treatment induced these p53 modifications. In Fig. 4C immunoprecipitates obtained by anti-p53 antibodies followed by Western analysis of its phosphorylated and acetylated status demonstrate that 4-24 h of treatment induced a marked increase in phospho-ser15-p53, and acetyl-Lys-320 and-Lys373-p53. This suggests that PTX treatment induced p53 posttranslational modification and that these modification could be responsible for 553 stabilization and trapping in the nuclei. The results also show (Fig. 4D) that PTX increased both, $\mathrm{p} 21^{\mathrm{WAF} 1}$ protein and mRNA levels. This, together with the increases observed in MDM2 levels, strongly suggests that p53 is transcriptionally active.

Involvement of FasR/Fas/L and JNK in PTX-induced apoptosis. As drugs that disturb microtubule dynamics can kill cells in a FasR/FasL-dependent manner, we examined if this pathway could be implicated in PTX-induced apoptosis in Y79 cells. In Fig. 5A RT/PCR analysis shows that FasR mRNA progressively increased between $0.5-2 \mathrm{~h}$ of PTX treatment, then the level was stable until $8 \mathrm{~h}$ of treatment, after which (16-24 h) the value decreased to the basal levels. Also FasL was up-regulated by PTX treatment, with mRNA levels markedly increasing between 0.5 and $8 \mathrm{~h}$ of treatment and then decreasing again to the basal values from 16 to $24 \mathrm{~h}$ of treatment. Western blot analysis (Fig. 5B) shows that after PTX treatment FasR and FasL proteins markedly increased exhibiting high molecular weight forms, likely reflecting intense oligomerization and activation of both the receptor and the ligand, which suggest the presence of active and stable aggregates, probably due to N-linked glycosylation or palmitoylation (Fig. 5B). The results also suggest (Fig. 5C) that FasR/FasL interact, as immunoprecipitate obtained with anti-FasR antibody markedly reacted with FasL. We also studied whether the activation of FasR/FasL was accompanied by JNK activation. Fig. 5D shows that JNK increased with PTX treatment which also induced its activation by phosphorylation. JNK activation was also suggested by the results which show c-Jun phosphorylation after PTX treatment.

In Fig. 5E experiments performed by immunoprecipitation with anti-JNK antibodies, suggests that JNK can associate with p53 as PTX significantly increases JNK/p53 complex which also displayed the presence of phospho-ser-15 p53.

Involvement of Bcl-2 family proteins and mitochondria in PTX-induced apoptosis. Since PTX-activation of JNK is often associated with Bcl-2 phosphorylation/inactivation, we evaluated the status of $\mathrm{Bcl}-2$ protein. Fig. $6 \mathrm{~A}$ shows that Y79 cells express very high levels of Bcl-2 which, after PTX treatment showed the appearance of a slow-mobility form which suggested Bcl-2 phosphorylation. Phosphorylation was evident at 2-h treatment, peaked at 16 and $24 \mathrm{~h}$ and markedly lowered after $48 \mathrm{~h}$. Treatment of Y79 cells with 
A

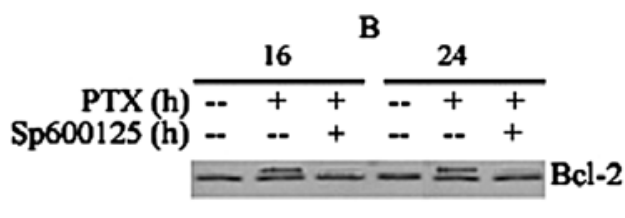

PTX (h) $-\begin{array}{lllllllll}2 & 2 & 4 & 8 & - & 16 & 24 & 48\end{array}$



C

PTX (h) $=-8 \quad 8 \quad 16 \quad 24$

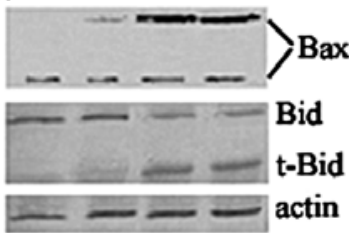

D

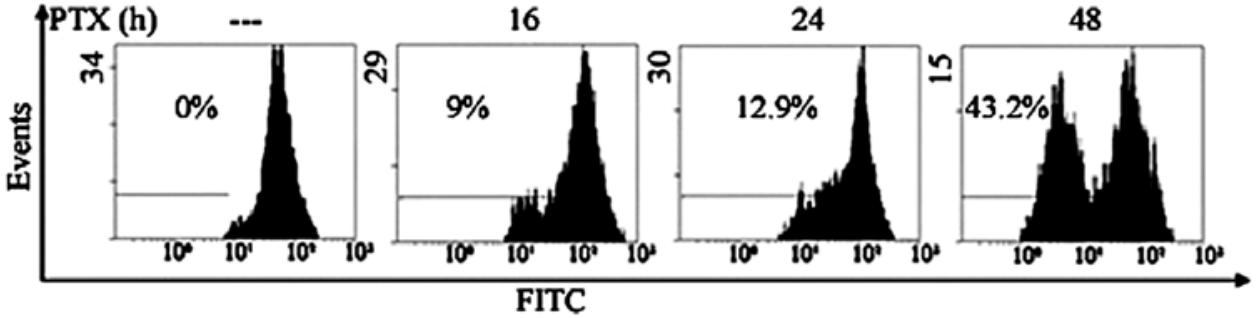

Figure 6. Involvement of Bcl-2 family and mitochondria in PTX induced apoptosis. Y79 cells were treated with 5 nM PTX for the indicated times and examined as described in Materials and methods by: Western blotting (A, B and C) and flow cytometry analysis (D) to evaluate the involvement of mitochondria in apoptosis induced by PTX treatment. The results obtained with SP600125 (B), a specific inhibitor of JNK, suggest the involvement of JNK in Bcl-2 phosphorylation. The results reported are representative of at least four independent experiments.

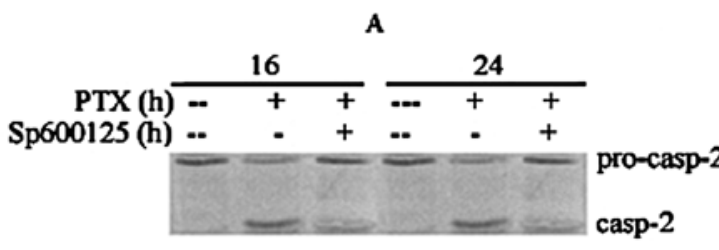

c
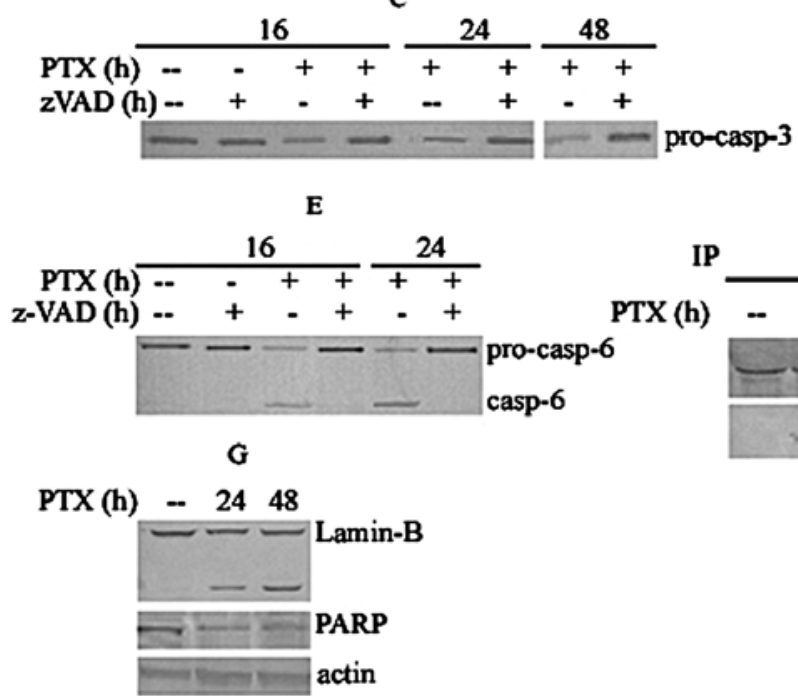

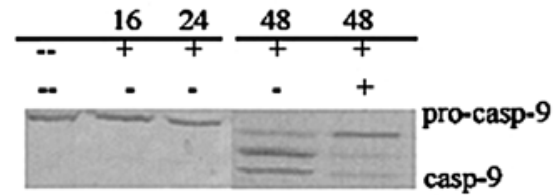

PTX (h) $=24$

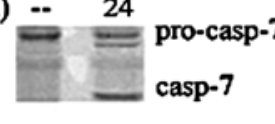

IP cyt C whole extracts

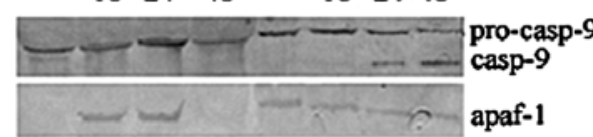

apaf-1

Figure 7. Involvement of caspase pathway in paclitaxel-induced apoptosis. Y79 cells were treated with $5 \mathrm{nM}$ PTX for the indicated times and examined as described in Materials and methods by: (A, B, C, D, E and G) Western blot analysis to describe the caspase family with its substrates; (F) immunoprecipitation analysis to describe the caspase-9/apaf-1 interaction. The results reported are representative of at least five independent experiments.

$20 \mu \mathrm{M}$ SP600125, a specific reversible ATP-competitive inhibitor of JNK (Fig. 6B), markedly decreased the effects induced by PTX on Bcl-2 phosphorylation. Fig. 6C shows that 16-24-h of PTX treatment potently increased bax levels 
inducing the presence of molecular forms of Bax at high molecular weight $(\sim 80 \mathrm{kDa})$ which suggests the presence of Bax multimers. At the same time, PTX caused the reduction of full-length Bid with the production of the active truncated t-Bid-form.

The study of mitochondrial membrane potential $\left(\Delta \psi_{\mathrm{m}}\right)$, using the ampholytic cationic fluorochrome $\mathrm{DiOC}_{6}$ and flow cytometry analysis (Fig. 6D), shows that PTX induced a significant decrease in $\mathrm{DiOC}_{6}$ uptake, indicating a loss of $\Delta \psi_{\mathrm{m}}$. The membrane potential progressively decreased by 9 , 13 and $43 \%$ at 16, 24 and 48 h respectively, thus demonstrating that PTX disturbed mitochondrial functions leading to $\Delta \psi_{\mathrm{m}}$ disruption at time points which were in good agreement with apoptosis activation.

Involvement of caspases in paclitaxel-induced apoptosis in Y79 cells. Next we investigated whether initiator and executioner caspases are activated during PTX-induced apoptosis. Fig. 7A shows that procaspase-2 significantly decreased after PTX treatment with the appearance of its processed/active fragment. Also the $\sim 52-\mathrm{kDa}$ proform of caspase-9 diminished in the course of PTX treatment (Fig. 7B) generating its active forms ( 35 and $37 \mathrm{kDa}$ ) which, in turn, will activate executioner caspases. After 16-48 h of PTXtreatment the levels of the $32 \mathrm{kDa}$ procaspase- 3 markedly decreased (Fig. 7C), although the expected increases of the 17- and 10-kDa active forms were not observed. PTX also activated caspase-7, as suggested by the decrease of the fulllength $35-\mathrm{kDa}$ procaspase-7 and the formation of both the 32-kDa partially activated form and the p19 fully processed form (Fig. 7D); the progressive activation of caspase-6, with the processing of the $34-\mathrm{kDa}$ procaspase form into the active 13-kDa fragment (Fig. 7E) is shown. To study whether the breach of mitochondrial membrane obtained after PTX treatment allowed for the release of apoptogenic factors from mitochondria and the apoptosome formation, immunoprecipitate obtained by anti-cytochrome c antibody were reacted with both anti-Apaf-1 and anti-procaspase- 9 antibodies (Fig. 7F). The figure shows that cyt $\mathrm{C}$ potently interacted with each other, thus suggesting the formation of apoptosomes. Fig. 7G shows that after 24 and $48 \mathrm{~h}$ of PTX treatment, the time when apoptosis was maximally evidenced, the activation of caspases was accompanied by the cleavage of lamin B from the $67-\mathrm{kDa}$ full-length form to the $45-\mathrm{kDa}$ fragment. A potent reduction in the levels of the $116-\mathrm{kDa}$ full-length form of PARP was also observed, but neither the $85-\mathrm{kDa}$ nor the 24-kDa fragments were evidenced. The use of the cell-permeable, irreversible pan-caspase inhibitors Z-VADFMK, caused significant inhibition of PTX-induced cleavage of caspase-3 and -6 (Fig. 7C and E). Furthermore, incubation with SP600125, the specific inhibitor of JNK, partially suppressed caspase-2 and -9 activation (Fig. 7A and B).

\section{Discussion}

In this study we demonstrate that, in human retinoblastoma Y79 cells, very low doses of PTX $(5 \mathrm{nM})$ potently induces $\mathrm{G}_{2} / \mathrm{M}$ phase arrest followed by apoptosis. The most relevant result suggests a scenario where E2F-1 plays a critical role in the effects induced by PTX. This, to our knowledge, is quite a novelty. FasR/FasL, JNK, p53 and Bcl-2 family, with mitochondrial pathways and the involvement of caspase cascades are implicated.

Many cells exposed to Taxol exhibit disruption of microtubule function with block of cell-cycle progression at the mitotic checkpoint (28). Mitotic block may persist for varying times and this can lead to overinduction of upstream events, including cyclin accumulation (29). Then, depending on the cell type, cells can either exit mitosis re-entering cell cycle without cell division (mitotic slippage) or undergo apoptosis. In many types of cancer cells pRb-regulated mechanisms are disrupted and this can be accompanied by E2F-1 overexpression with inappropriate upregulation of cyclins (30), start of S-phase and apoptosis. Our results demonstrating that PTX induces potent $\mathrm{G}_{2}-\mathrm{M}$ arrest, are highly suggestive of interference by PTX with spindle formation, as the evidence of long thick microtubule bundles surrounding the nucleus showed. In Y79 cells we have found significant basal levels of E2F1 which potently increased with PTX treatment. This was accompanied by a progressive increase in cyclin $\mathrm{E}$, cyclin A and Cyclin B1 throughout the treatment, and potent apoptosis. Thus, the E2F1-induced accumulation of cyclins could, in part, account for the activation of apoptosis. It seems that E2F1 is part of an anti-tumor safeguard mechanism that engages cell-death pathways to protect organisms from the development of tumors (31). This may be particularly interesting to oncologists, as deregulation of E2F1 activity occurs in the majority of human tumors and in tumors that have not completely disabled the apoptotic pathway this apoptosis sensitization may provide the 'therapeutic window' which would allow chemotherapy to selectively kill cancer cells.

In this study, that paclitaxel kills Y79 cells through the induction of apoptosis is shown by morphological, biochemical, and molecular criteria; that such an event would result from $\mathrm{G}_{2} / \mathrm{M}$ arrest, is evidenced by cell cycle analysis which shows that $\mathrm{G}_{2} / \mathrm{M}$ arrest significantly preceded apoptosis. PTX also induced potent modification in the functional status of $\mathrm{Cdc} 2$, protein which when complexed with cyclin B1 represents the major player of the $G_{2} / M$ checkpoint. This checkpoint, if inappropriately activated, can lead to catastrophic premature mitosis and apoptosis (32). Here we show that after 4-8 h of treatment when the cells accumulated in $\mathrm{G}_{2}-\mathrm{M}$ phase and apoptosis was almost absent, most of the $\mathrm{Cdc} 2$ protein was in the phosphorylated (inactive) form; after 16-48 h of treatment, when potent apoptotic events were shown, the phosphorylated (inactive) form of $\mathrm{Cdc} 2$ potently diminished. Taken together, our results suggest that in Y79 cells such a late activation of Cdc2 might be responsible for a mitotic catastrophe which stimulates the apoptotic program (33). We have shown that PTX induces an early and prolonged increase in p53 expression and its stabilization and nuclear translocation. Consistently, our data also show that PTX increased $\mathrm{p} 21^{\mathrm{WAF} 1} \mathrm{mRNA}$ and protein levels, suggesting that p53 is transcriptionally active. The increase in p53 seems to correlate with cell cycle arrest until $16 \mathrm{~h}$ of treatment. Then, after 16-48 $\mathrm{h}$ of treatment both the increase in p53 levels and its nuclear translocation correlated well with cell death, as is also suggested by experiments performed with p53-inhibitor PFT- $\alpha$. The increase in p53 level induced by 
PTX treatment was accompanied by a potent p53-Lys-320 and -373-acetylation and ser-15-phosphorylation which account for p53 stabilization. Phospho-ser-15 is essential for the transactivation of p53 and for the inhibition of p53 binding by MDM2 which, targeting p53 for rapid ubiquitinmediated proteosomal degradation, accounts for the normally short half-life of p53. PTX increased MDM2 levels, and this accounts for p53 activity, but it simultaneously decreases MDM2/p53 interaction while increasing both p14 ${ }^{\mathrm{ARF}}$ (a protein that inactivate MDM-2, and activates p53-dependent transcription and p53 stabilization) and its interaction with MDM2. The $\mathrm{p} 14^{\mathrm{ARF}} / \mathrm{p} 53 / \mathrm{Mdm} 2$ pathway serves as a checkpoint that protects cells from oncogene-induced transformation.

As both E2F-1 and p14 ${ }^{\mathrm{ARF}}$ increased after $30 \mathrm{~min}$ of PTX treatment, while p53 was induced after $2 \mathrm{~h}$ of PTX treatment, our results suggest that p53 accumulates following E2F1 expression through activation of p14 ${ }^{\mathrm{ARF}}$, which in turn interacts with MDM2, thereby preventing p53 targeting for degradation. The results underscore the importance of E2F1 as a possible key mediator of sensitivity to PTX.

Interestingly, E2F1 has been also implicated in Fasinduced apoptosis by increasing Fas signalling $(34,35)$. In our study RT/PCR and Western blot analysis show progressive increases in the expression of FasL and FasR which, like E2F1, start 30 min after PTX treatment. In Y79 cells FasR/ FasL seem to signal through the JNK pathway, since both JNK levels and its activation by phosphorylation increased by PTX treatment. Intriguingly, 16-48 h of PTX treatment resulted in a dramatic Bcl-2 phosphorylation/inactivation. Since the Bcl-2 protein is important for suppression of mitochondrial manifestations of apoptosis (36), this suggests that JNK activation, by inactivating Bcl-2, may activate apoptosis, as indicated by treatment with SP600125, a specific competitive inhibitor of JNK, which inhibited both Bcl-2 phosphorylation and apoptosis. Importantly, the dramatic phosphorylation of Bcl-2 induced by PTX was accompanied by Bid fragmentation and a significant increase of Bax expression, thus suggesting that changes in the ratio of antiapoptotic/proapoptotic Bcl-2 family might contribute to the apoptosis-promoting PTX activity throughout $\Delta \psi_{\mathrm{m}}$ disruption. In a number of tumors, enforced E2F1 expression results in the accumulation of initiator and executioner caspases, through p53-dependent signals. The activation of caspase-cascade can occur through either the activation of the death receptors, or the activation of mitochondrial pathway. Crosstalk between the two apoptosis pathways occurs when initiator caspases, activated by FasR, convert and activate Bid into t-Bid that interacts with Bax and induces the loss of mitochondrial transmembrane potential (37). Here, we report that PTX treatment is accompanied by activation of caspase- 2 and accumulation of t-Bid which suggest a connection between Fas activation and Bid truncation. We also show the activation of apoptosomes and of caspases-3, -6 and-7 accompanied by the cleavage of lamin B and the decreased levels of PARP. The use of the irreversible pan-caspase inhibitors Z-VADFMK, and the incubation with the selective JNK inhibitor SP600125, significantly inhibited PTX-induced cell death and partially suppressed activation of caspases and lamin-B fragmentation.
In conclusion, in Y79 cells PTX-induced apoptosis offers a scenario where E2F1 seems to be a principal player. Both intrinsic and extrinsic pathways seemed to be involved, probably resulting from E2F1/Fas/JNK/caspase-2 activation, with p53, Bcl2, Bax and t-Bid designated to be the main protagonists of the apoptotic event. In turn, activated Bid can mediate mitochondrial apoptotic pathways with loss of mitochondrial potential and activation of a caspase cascade and consequent cell death.

Taxanes are used for the treatment of many human cancers, as first- and second-line chemotherapeutics (38). Paclitaxel has a range of activity that includes ovarian cancer, melanoma, lung carcinoma and other tumors (39). This study suggests to consider PTX for clinical investigation in patient with retinoblastoma. Our laboratory is currently working to silence either E2F1 or p53 in Y79 cells.

\section{Acknowledgements}

This study was supported by grants from the Italian Ministry of University and Research (MUR).

\section{References}

1. Melamud A, Palekar R and Singh A: Retinoblastoma. Am Fam Physician 73: 1039-1044, 2006.

2. Chintagumpala M, Chevez-Barrios P, Paysse EA, Plon SE and Hurwitz R: Retinoblastoma: review of current management. Oncologist 12: 1237-1246, 2007.

3. Antoneli CB, Ribeiro KC, Steinhorst F, Novaes PE, Chojniak MM and Malogolowkin M: Treatment of retinoblastoma patients with chemoreduction plus local therapy: experience of the AC Camargo Hospital, Brazil. J Pediatr Hematol Oncol 28: 342-345, 2006.

4. Lambert MP, Shields C and Meadows AT: A retrospective review of hearing in children with retinoblastoma treated with carboplatin-based chemotherapy. Pediatr Blood Cancer 50: 223-226, 2007.

5. Goodrich DW: The retinoblastoma tumor-suppressor gene, the exception that proves the rule. Oncogene 25: 5233-5243, 2006.

6. Sellers WR and Kaelin WG Jr: Role of the retinoblastoma protein in the pathogenesis of human cancer. J Clin Oncol 15: 3301-3312, 1997.

7. Giacinti $\mathrm{C}$ and Giordano $\mathrm{A}: \mathrm{RB}$ and cell cycle progression. Oncogene 25: 5220-5227, 2006.

8. Chakravarti A, Heydon K, Wu CL, Hammond E, Pollack A, Roach M, Wolkov H, Okunieff P, Cox J, Fontanesi, Abrams R, Pilepich M and Shipley W: Loss of p16 expression is of prognostic $\mathrm{J}$ significance in locally advanced prostate cancer: an analysis from the radiation therapy oncology group protocol 86-10. J Clin Oncol 21: 3328-3334, 2003.

9. De Blasio A, Messina C, Santulli A, Mangano V, Di Leonardo E, D'Anneo A, Tesoriere G and Vento R: Differentiative pathway activated by 3-aminobenzamide, an inhibitor of PARP, in human osteosarcoma MG-63 cells. FEBS Lett 579: 615-620, 2005.

10. Emanuele S, D'Anneo A, Bellavia G, Vassallo B, Lauricella M, De Blasio A, Vento R and Tesoriere G: Sodium butyrate induces apoptosis in human hepatoma cells by a mitochondria/caspase pathway, associated with degradation of beta-catenin, $\mathrm{pRb}$ and Bcl-XL. Eur J Cancer 40: 1441-1452, 2004.

11. De Blasio A, Musmeci MT, Giuliano M, Lauricella M, Emanuele S, D'Anneo A, Vassallo B, Tesoriere G and Vento R: The effect of 3-aminobenzamide, inhibitor of poly(ADP-ribose) polymerase, on human osteosarcoma cells. Int J Oncol 23: 1521-1528, 2003.

12. Lauricella M, Calvaruso G, Carabillò M, D'Anneo A, Giuliano M, Emanuele S, Vento R and Tesoriere G: pRb suppresses camptothecin-induced apoptosis in human osteosarcoma Saos-2 cells by inhibiting c-Jun N-terminal kinase. FEBS Lett 499: 191-197, 2001 . 
13. Giuliano M, Lauricella M, Vassallo E, Carabillò M, Vento R and Tesoriere G: Induction of apoptosis in human retinoblastoma cells by topoisomerase inhibitors. Invest Ophthalmol Vis Sci 39: 1300-1311, 1998

14. Lauricella M, D'Anneo A, Giuliano M, Calvaruso G, Emanuele S, Vento R and Tesoriere G: Induction of apoptosis in human osteosarcoma Saos- 2 cells by the proteasome inhibitor MG132 and the protective effect of $\mathrm{pRb}$. Cell Death Differ 10: 930-932, 2003.

15. Di Felice V, Lauricella M, Giuliano M, Emanuele S, Vento R and Tesoriere G: The apoptotic effects of cisplatin and carboplatin in retinoblastoma Y79 cells. Int J Oncol 13: 225-232, 1998.

16. Vento R, D'Alessandro N, Giuliano M, Lauricella M, Carabillò M and Tesoriere G: Induction of apoptosis by arachidonic acid in human retinoblastoma Y79 cells: involvement of oxidative stress. Exp Eye Res 70: 503-517, 2000.

17. Vento R, Giuliano M, Lauricella M, Carabillò M, Di Liberto D and Tesoriere G: Induction of programmed cell death in human retinoblastoma Y79 cells by C2-ceramide. Mol Cell Biochem 185: 7-15, 1998.

18. Giuliano M, Lauricella M, Calvaruso G, Carabillò M, Emanuele S, Vento R and Tesoriere G: The apoptotic effects and synergistic interaction of sodium butyrate and MG132 in human retinoblastoma Y79 cells. Cancer Res 59: 5586-5595, 1999.

19. Lauricella M, Calvaruso G, Giuliano M, Carabillò M, Emanuele $S$, Vento R and Tesoriere G: Synergistic cytotoxic interactions between sodium butyrate, MG132 and camptothecin in human retinoblastoma Y79 cells. Tumour Biol 21:337-348, 2000.

20. Calvaruso G, Carabillò M, Giuliano M, Lauricella M, D'Anneo A, Vento R and Tesoriere G: Sodium phenylbutyrate induces apoptosis in human retinoblastoma Y79 cells: the effect of combined treatment with the topoisomerase I-inhibitor topotecan. Int J Oncol 18: 1233-1237, 2001

21. Marupudi NI, Han JE, Li KW, Renard VM, Tyler BM and Brem H: Paclitaxel: a review of adverse toxicities and novel delivery strategies. Expert Opin Drug Saf 6: 609-621, 2007.

22. Wang J, He FT, Tzang CH, Fong WF, Xiao PG, Han R and Yang MS: Differential gene expression profiles in paclitaxelinduced cell cycle arrest and apoptosis in human breast cancer MCF-7 cells. Yao Xue Xue Bao 40: 1099-1104, 2005.

23. Lin ZZ, Hsu C, Chang YC, Yu CJ, Hsu CH, Lin CC, Cheng AL, Yang PC and Yang CH: Outcome of advanced non-small cell lung cancer patients receiving gemcitabine and weekly paclitaxel as first-line treatment. Lung Cancer (In press).

24. Kawai H, Lopez-Pajares V, Kim MM, Wiederschain D and Yuan Z-M: RING domain-mediated interaction is a requirement for MDM2's E3 ligase activity. Cancer Res 67: 6026-6030, 2007.

25. Chène P: Inhibition of the p53-MDM2 interaction: targeting a protein-protein interface. Mol Cancer Res 2: 20-28, 2004.
26. Lowry OH, Rosebrough NJ, Farr AL and Randall RJ: Protein measurement with the Folin phenol reagent. J Biol Chem 193: 265-275, 1951.

27. Mosmann T: Rapid colorimetric assay for cellular growth and survival: application to proliferation and cytotoxicity assays. J Immunol 32: 671-676, 1983.

28. Horwitz SB: Mechanism of action of taxol. Trends Pharmacol Sci 13: 131-136, 1992.

29. Perez-Stable C: 2-Methoxyestradiol and paclitaxel have similar effects on the cell cycle and induction of apoptosis in prostate cancer cells. Cancer Lett 231: 49-64, 2006.

30. Kaelin WG Jr: E2F1 as a target: promoter-driven suicide and small molecule modulators. Cancer Biol Ther 2: S48-S54, 2003.

31. Hershko T, Chaussepied M, Oren M and Ginsberg D: Novel link between E2F and p53: proapoptotic cofactors of p53 are transcriptionally upregulated by E2F. Cell Death Differ 12: 377-383, 2005.

32. Stanelle J and Pützer BM: E2F1-induced apoptosis: turning killers into therapeutics. Trends Mol Med 12: 177-185, 2006.

33. Xiao Z, Xue J, Semizarov D, Sowin TJ, Rosenberg SH and Zhang H: Novel indication for cancer therapy: Chk1 inhibition sensitizes tumor cells to antimitotics. Int J Cancer 115: 528-538, 2005.

34. Stumm S, Meyer A, Lindner M, Bastert G, Wallwiener D and Gückel B: Paclitaxel treatment of breast cancer cell lines modulates Fas/Fas ligand expression and induces apoptosis which can be inhibited through the CD40 receptor. Oncology 66: 101-111, 2004.

35. Cao Q, Xia Y, Azadniv M and Crispe IN: The E2F-1 transcription factor promotes caspase- 8 and bid expression, and enhances Fas signaling in T cells. J Immunol 173: 1111-1117, 2004

36. Cory S, Huang DC and Adams JM: The Bcl-2 family: roles in cell survival and oncogenesis. Oncogene 22: 8590-8607, 2003.

37. Basu A, Castle PV, Bouziane M, Bhalla K and Haldar S: Crosstalk between extrinsic and intrinsic cell death pathways in pancreatic cancer: synergistic action of estrogen metabolite and ligands of death receptor family. Cancer Res 66: 4309-4318, 2006.

38. Braunhut SJ, McIntosh D, Vorotnikova E, Zhou T and Marx KA Detection of apoptosis and drug resistance of human breast cancer cells to taxane treatments using quartz crystal microbalance biosensor technology. Assay Drug Dev Technol 3: $77-88,2005$.

39. Foa R, Norton L and Seidman AD: Taxol (paclitaxel): a novel anti-microtubule agent with remarkable anti-neoplastic activity. Int J Clin Lab Res 24: 6-14, 1994. 